\title{
Use of angiotensin-converting enzyme inhibitors and freedom from amputation after lower extremity revascularization
}

This article was published in the following Dove Press journal:

Vascular Health and Risk Management

14 July 2017

Number of times this article has been viewed

\author{
Jared E Kray' \\ Viktor Y Dombrovskiy² \\ Todd R Vogel' \\ 'Department of Surgery, Division of \\ Vascular Surgery, School of Medicine, \\ University of Missouri, Columbia, MO, \\ ${ }^{2}$ Department of Surgery, Rutgers- \\ Robert Wood Johnson Medical School, \\ New Brunswick, NJ, USA
}

Objective: Angiotensin-converting enzyme inhibitors (ACEIs) have not been well evaluated in conjunction with lower extremity revascularization (LER). This study evaluated freedom from amputation in patients who underwent either an open (OPEN) or endovascular (ENDO) revascularization with and without utilization of an ACEI.

Materials and methods: Patients who underwent LER were identified from 2007-2008 Medicare Provider Analysis and Review files. Demographics, comorbidities, and disease severity were obtained. Post-procedural use of an ACEI was confirmed using combining them with National Drug Codes and Part D Files. Outcomes were analyzed using chi-square analysis, Kaplan-Meier test, and Cox regression.

Results: We identified 22,954 patients who underwent LER: 8,128 (35.4\%) patients with claudication, 3,056 (13.3\%) with rest pain, and 11,770 (51.3\%) with ulceration or gangrene. More patients underwent ENDO $(14,353)$ than $\operatorname{OPEN}(8,601)$ revascularization and $38 \%$ of the cohort was taking an ACEI. Overall, ACEI utilization compared to patients not taking ACEI was not associated with lower amputation rates at 30 days (13.5\% vs. $12.6 \%)$, 90 days $(17.7 \%$ vs. $17.1 \%)$, or 1 year ( $23.9 \%$ vs. $22.8 \%)(P>0.05$ for all). After adjustment for comorbidities, ACEI utilization was associated with higher amputation rates for patients with rest pain (hazard ratio: 1.4 ; $95 \%$ confidence interval: $1.1-1.8)$.

Conclusion: ACEI utilization was not associated with overall improved rates of amputation-free survival or overall survival in the vascular surgery population. However, an important finding of this study was that patients presenting with a diagnosis of rest pain and taking an ACEI who underwent a LER had statistically higher amputation rates and a lower amputation-free survival at 1 year. Further analysis is needed to delineate best medical management for patients with critical limb ischemia and taking ACEI who undergo vascular revascularization.

Keywords: angiotensin-converting enzyme inhibitor, lower extremity revascularization, amputation

\section{Introduction}

Peripheral arterial disease (PAD) is estimated to affect the lives of over 8 million people in the USA. ${ }^{1}$ In patients older than 60 years, $\sim 12 \%-20 \%$ have signs or symptoms of peripheral vascular disease. ${ }^{2}$ Commonly associated comorbid conditions frequently seen in patients with PAD are tobacco abuse, hypertension, diabetes, dyslipidemias, and other atherosclerotic manifestations such as coronary artery disease. In general, intervention in patients is reserved for patients who can be subcategorized by symptoms: claudication, rest pain, or tissue loss/gangrene.

Angiotensin-converting enzyme inhibitors (ACEIs) were originally intended for the management of hypertension. Early experience with these drugs demonstrated benefits
Correspondence: Todd R Vogel Department of Surgery, Division of Vascular Surgery, School of Medicine, University of Missouri, One Hospital Drive, Columbia, MO 65212, USA

Tel +l 573882 I308

Fax +I 5738845049

Email vogeltr@health.missouri.edu 
in a wide range of patients and then expanded indications for patients with diabetic nephropathy, as well as a positive effect on cardiac patients showing improved remodeling of heart tissue. $^{3}$ Some studies have even shown all-cause mortality, stroke, and myocardial infarction to be lower in any patient with left ventricular dysfunction even without heart failure symptoms. ${ }^{4}$ This has led a large number of PAD patients undergoing lower extremity revascularization (LER) to take these medications.

To date, very few have looked at the correlation of ACEIs and outcomes after LER. The objective of this analysis was to evaluate the outcomes in patients under ACEI medications and who underwent LER.

\section{Materials and methods}

Using the Centers for Medicare \& Medicaid Services (CMS) files for the years 2007-2008 - Medicare Provider Analysis and Review (MedPAR) File, Carrier Claim File, Part D Drug Event (PDE) File, and Beneficiary Summary File - we identified patients aged 65 years and older who underwent LER. These files contain information from inpatient hospital records including sociodemographic characteristics, International Classification of Diseases - Ninth Revision, Clinical Modification (ICD-9-CM) diagnosis codes, Current Procedural Terminology, Fourth Edition (CPT-4), codes for all procedures during hospitalization, hospital length of stay and cost, patient outcomes as discharge, and others. The PDE file includes information about all prescription drugs for Medicare beneficiaries such as National Drug Code (NDC) in the NDC11 format to identify drugs and their dosage, dispensed quantity and supply, and drug cost. All these data collected were then linked by a unique personal identifier to each Medicare beneficiary through CMS data.

Patient who underwent LER in 2007 and 2008 years were identified by CPT codes 35556, 35583, 35656, 35566, $35585,35666,35556,35583,35226,35256$, and 35286 for open (OPEN) revascularization and 35473, 35474, and 35470 for endovascular (ENDO) revascularization. Among them, we selected those with Part D coverage during the whole year. Using the ICD-9-CM diagnosis codes, these patients were allocated to one of the three groups according to their PAD severity: claudication, 440.21; rest pain, 440.22; and ulceration and gangrene, 440.23 and 440.24 . We then used 1,424 NDC11 codes in the PDE file and date of procedure in the Carrier Claim File to identify those patients who received ACEI prior to procedures. The study population who underwent amputation was then tracked using CPT codes 27590 , 27591, 27592, 27880, 27881, 27882, 28800, 28805, 28810, 28820 , and 28825 . Comorbidities were then evaluated using the Agency for Healthcare Research and Quality Healthcare Cost and Utilization Project comorbidity software (http:// www.hcup-us.ahrq.gov) applied to the MedPAR file.

\section{Statistical analysis}

Statistical analysis was carried out using SAS software, version 9.2 (SAS Institute, Cary, NC, USA). Chi-square analysis was used for comparison of categorical variables with calculating odds ratio (OR) and $95 \%$ confidence interval (CI). Results of bivariate analysis were then tested using multivariable logistic regression analysis with adjustment for age, gender, race, use of ACEI, disease severity, type of surgical procedure, and comorbidities. Student's $t$-test was used for comparison of continuous variables. Differences in 30-day, 90-day, and 1-year freedom from amputation survival with and without use of ACEI were analyzed using Kaplan-Meier curves and Cox proportional hazards regression models with the same adjustment. A $P$-value $\leq 0.05$ was considered statistically significant. The study was exempted by the University of Missouri Institutional Review Board as all of the data used were deidentified and offered no risk to patients. Informed patient consent was not required as the databases is completely deidentified using methods that are compliant with the Health Insurance Portability and Accountability Act (HIPAA).

\section{Results}

We identified 22,954 patients who underwent LER during the study period using the aforementioned methods. ENDO revascularization procedures were performed in $62.5 \%$ of all patients and OPEN procedures in $37.5 \%$ patients. The mean age of the cohort was 75.9 years. As shown in Table 1, the oldest patients were in the ulceration/gangrene group. Patients with rest pain were younger $(P=0.0003)$ and those with claudication had the smallest mean age $(P<0.0001)$. There were significant differences in gender and race structure between the three groups $(P<0.0001)$.

Common comorbid conditions were congestive heart failure (CHF), hypertension, chronic pulmonary disease, diabetes, renal failure, and obesity. These risk factors did vary depending upon the severity of PAD. Patients with ulceration/gangrene compared to their counterparts with rest pain or claudication had a higher prevalence of $\mathrm{CHF}$ $(P<0.0001$ for both), diabetes $(P<0.0001)$, and renal failure $(P<0.0001)$. However, patients with rest pain had higher incidence of chronic lung disorders than those with claudication $(P=0.0007)$ or ulceration/gangrene $(P<0.0001)$.

A total of 8,739 (38.1\%) patients received ACEIs prior to intervention. There were no statistically significant dif- 
Table I Patient demographics

\begin{tabular}{|c|c|c|c|c|}
\hline \multirow[b]{2}{*}{ Patient characteristics } & \multicolumn{3}{|l|}{ Indications } & \multirow[b]{2}{*}{ Total } \\
\hline & Claudication & Rest pain & Ulceration/gangrene & \\
\hline Age, years (mean \pm SD) & $74.7 \pm 6.4$ & $76.1 \pm 7.5$ & $76.7 \pm 8.4$ & $75.9 \pm 7.7$ \\
\hline \multicolumn{5}{|l|}{ Age groups, years } \\
\hline $65-69$ & 2,178 (26.8\%) & 737 (24.1\%) & 3,027 (25.7\%) & 5,942 \\
\hline 70-74 & $2,112(26.0 \%)$ & $655(21.4 \%)$ & $2,053(17.5 \%)$ & 4,820 \\
\hline 75-79 & $\mathrm{I}, 853(22.8 \%)$ & $636(20.8 \%)$ & 2,085 (I7.7\%) & 4,574 \\
\hline $80+$ & I,985 (24.4\%) & $\mathrm{I}, 028(33.7 \%)$ & $4,605(39.1 \%)$ & 7,618 \\
\hline \multicolumn{5}{|l|}{ Gender } \\
\hline Male & 4,008 (49.3\%) & I,3। 3 (43.0\%) & $5,388(45.8 \%)$ & 10,709 \\
\hline Female & $4,120(50.7 \%)$ & $1.743(57.0 \%)$ & $6,382(54.2 \%)$ & 12,245 \\
\hline \multicolumn{5}{|l|}{ Race } \\
\hline Whites & 6,759 (83.1\%) & 2,347 (76.8\%) & 8,549 (72.6\%) & 17,655 \\
\hline Blacks & $853(10.5 \%)$ & $521(17.0 \%)$ & $2,308(19.6 \%)$ & 3,682 \\
\hline Hispanics & $275(3.4 \%)$ & $113(3.7 \%)$ & $518(4.4 \%)$ & 906 \\
\hline Others and missing & $24 I(3.0 \%)$ & $75(2.5 \%)$ & 395 (3.4\%) & 711 \\
\hline \multicolumn{5}{|l|}{ Comorbidities } \\
\hline $\mathrm{CHF}$ & 347 (4.3\%) & 180 (5.9\%) & I,530 (I $3.0 \%)$ & 2,057 \\
\hline Hypertension & 6,171 (75.9\%) & $2,220(72.6 \%)$ & 7,331 (62.3\%) & 15,722 \\
\hline Chronic pulmonary disease & I,907 (23.5\%) & $811(26.5 \%)$ & $1,948(16,6 \%)$ & 4,666 \\
\hline Pulmonary circulation disease & $29(0.4 \%)$ & $16(0.5 \%)$ & $93(0.8 \%)$ & 138 \\
\hline Diabetes & $2,695(33.2 \%)$ & $\mathrm{I}, 08 \mathrm{I}(35.4 \%)$ & $5,040(42.8 \%)$ & 8,816 \\
\hline Renal failure & $898(11.1 \%)$ & 405 (13.2\%) & $3,156(26.8 \%)$ & 4,459 \\
\hline Obesity & $333(4.1 \%)$ & $88(2.9 \%)$ & $285(2.4 \%)$ & 706 \\
\hline \multicolumn{5}{|l|}{ ACEI before procedure } \\
\hline Yes & 3,065 (37.7\%) & I, I 89 (38.9\%) & 4,485 (38.1\%) & 8,739 \\
\hline No & $5,063(62.3 \%)$ & $\mathrm{I}, 867(6 \mathrm{I} .1 \%)$ & 7,285 (61.9\%) & 14,215 \\
\hline Total & $8,128(35.4 \%)$ & $3,056(13.3 \%)$ & $1 \mathrm{I}, 770(5 \mathrm{I} .3 \%)$ & 22,954 \\
\hline
\end{tabular}

Abbreviations: ACEl, Angiotensin-converting enzyme inhibitor; SD, standard deviation; CHF, congestive heart failure.

ferences in patients with claudication, rest pain, or tissue loss when evaluating ACEI usage. Thirty-day amputation crude rates in the whole study population were slightly different among groups who received and did not receive ACEI (Figure 1). Those on ACEI demonstrated $13.5 \%$ vs. $12.6 \%$ rate $(P=0.04)$ during 30 days after LER. By 90 days after LER, this significance disappeared (17.8\% vs. $17.1 \%$; $P=0.2$, respectively), but it was noticed again at the end of the year after procedure $(23.9 \%$ vs. $22.8 \%$, respectively; $P=0.04)$. However, the multivariable analysis after adjustment for age, gender, race, disease severity, type of surgical procedure, and comorbidities showed no significant

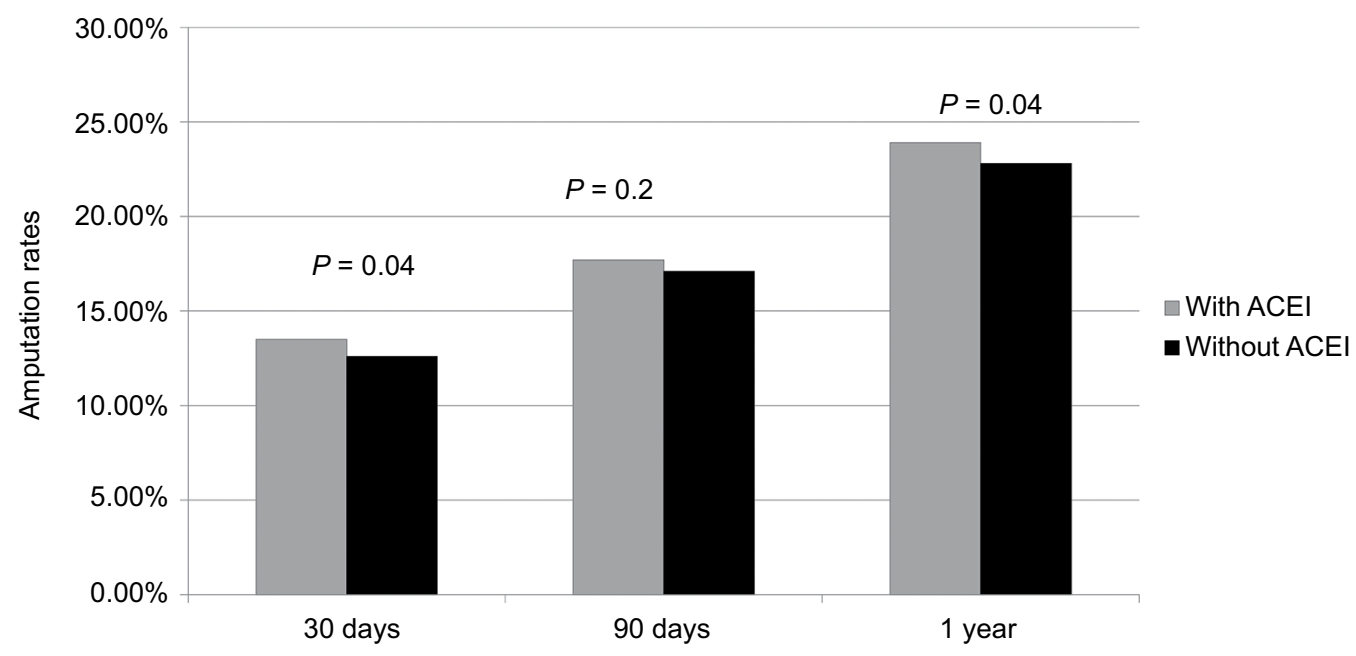

Figure I Crude amputation rates after LER with and without ACEls.

Abbreviations: LER, lower extremity revascularization; ACEls, angiotensin-converting enzyme inhibitors. 
differences. We then evaluated and compared amputation rates in patients who used and did not use ACEI depending on the severity of PAD. For claudication patients who underwent LER and who were on ACEI, 30-day, 90-day, and 1 year crude rates of amputation were $0.2 \%, 0.7 \%$, and $2.5 \%$, respectively. For those not taking ACEI, these rates were $0.4 \%, 1.0 \%$, and $2.3 \%$, respectively, and did not differ significantly. Multivariable logistic regression and survival analyses also showed no significant differences in this group.

LER for rest pain was associated with amputation rates of $1.93 \%$ at 30 days for patients who were either on or off of ACEI. Ninety-day amputation rates for this group were $4.8 \%$ for those on ACEI vs. 3.3\% $(P=0.04)$ for those who were not on ACEI. At 1 year, this difference persisted with an amputation rate of $10.2 \%$ for those on ACEI vs. $7.5 \%$ for those not on ACEI $(P<0.01)$. These results were confirmed by the multivariable analysis. Patients who did not receive ACEI compared to those who received ACEI before LER were less likely to have amputation by 90 days $(\mathrm{OR}=0.66$; 95\% CI $0.45-0.96 ; P=0.03)$ and 1 year after procedure $(\mathrm{OR}=0.71 ; 95 \%$ CI $0.55-0.93 ; P=0.01)$. Figure 2 demonstrates Kaplan-Meier curves for amputation-free survival of patients with rest pain during 1 year after LER. The Cox proportional hazards model showed that the hazard ratio (HR) of amputation during 1 year after LER in patients who received ACEI vs. non-receivers was equal to 1.37 with $95 \%$ CI equal to $1.07-1.76(P=0.01)$.

When patients underwent LER for ulceration or gangrene with ACEI before LER, the 30-day crude amputation rates were higher than those without ACEI (25.7\% vs. $23.8 \%$;
$P=0.02)$. This was confirmed by the multivariable logistic regression analysis $(\mathrm{OR}=1.1 ; 95 \% \mathrm{CI} 1.006-1.200 ; P=0.037)$. However, we could not find any similar difference by 90 days and 1 year after LER neither by the bivariate analysis (32.9\% vs. $31.8 \% ; P=0.22$ and $42.2 \%$ vs. $40.9 \% ; P=0.17$, respectively) nor by the multivariable analysis.

\section{Discussion}

This study represents a longitudinal analysis of US Medicare patients who underwent LER procedures. In this analysis, $38 \%$ of patients who underwent LER used an ACEI. Overall, after adjustment, ACEI utilization was not associated with poorer limb salvage rates. However, an important finding of this study is that patients with a diagnosis of rest pain who were on ACEI therapy and who underwent a LER had statistically higher amputation rates at 1 year compared to those who were not on ACEI therapy.

Studies have evaluated the effect of ACEI on patients with peripheral vascular diseases. Studies from the UK have looked specifically at ramipril and its effect on walking distance as well as quality of life. ${ }^{5}$ These authors concluded that among patients with intermittent claudication, 24-week treatment with ramipril resulted in significant increases in pain-free and maximum treadmill walking times compared with placebo. ${ }^{5}$ The results of these trials have been evaluated and two theories for this finding have been postulated. ACEIs are known to have positive effects on muscle remodeling after cardiac events and may also have effect on peripheral muscles. As well, ACEI are known to have effects on the endothelium, and their largest effect may be to decrease the stiffness of peripheral arteries. ${ }^{6,7}$ Despite

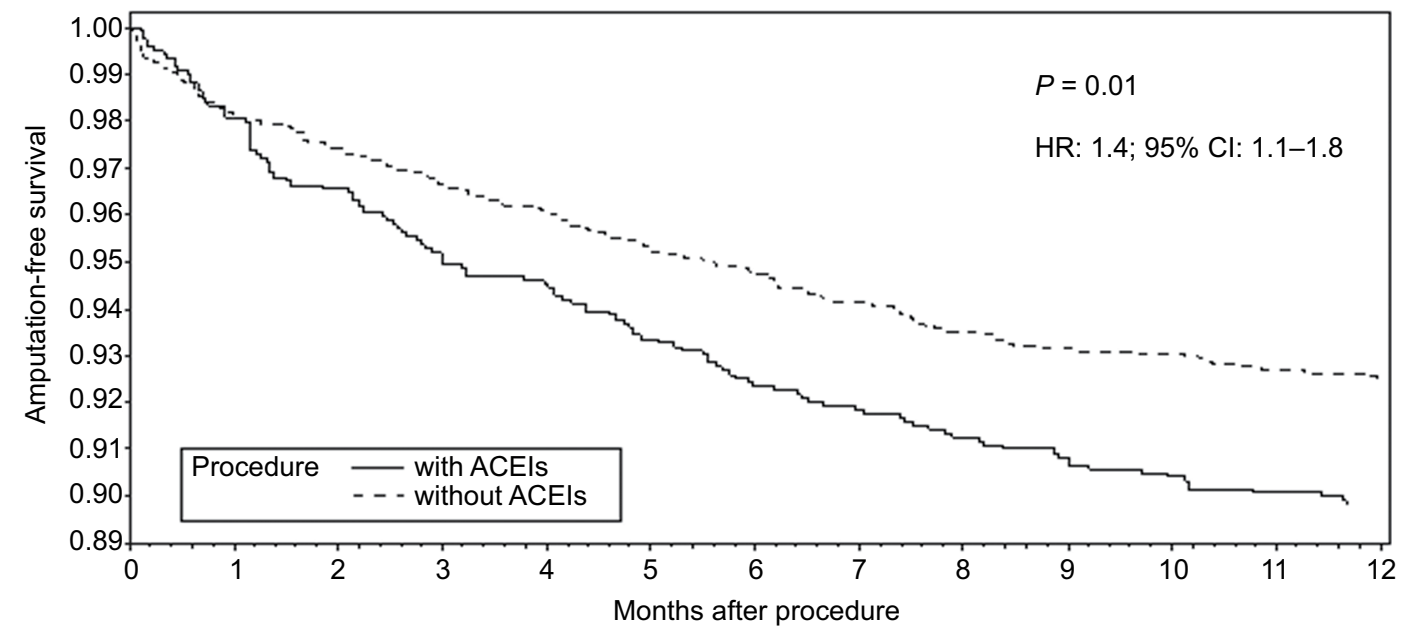

Figure 2 Amputation-free survival of patients with rest pain after LER with or without ACEls.

Abbreviations: ACEI, angiotensin-converting enzyme inhibitor; $\mathrm{Cl}$, confidence interval; $\mathrm{HR}$, hazard ratio; LER, lower extremity revascularization. 
these theorized benefits, another study was performed that evaluated vascular growth factors and increasing peripheral angiogenesis. ${ }^{8}$ Vascular endothelia growth factor (VEGF) has long been studied and it has been suggested that ACEI may inhibit VEGF and subsequent neovascularization or angiogenesis after revascularization. ${ }^{8}$ Therefore, one theory explaining the inferior outcomes in patients taking ACEI who had the diagnosis of rest pain may be that ACEI inhibited angiogenesis leading to more amputations over time. In this study, the effects of inhibited angiogenesis may be more pronounced or clinically significant in patients with critical limb ischemia (CLI) leading to increased amputation rates. Since PAD predominantly affects larger vessels, there may be no perceived benefit noted in the patients taking ACEIs as this analysis demonstrates. Further analysis is needed as these are suggestions for inferior outcomes in the ACEI group and randomized trials are need.

Hogh et al examined the association between ACEI use and clinical outcome after primary vascular reconstruction in a population-based follow-up study. ${ }^{9}$ They evaluated all Danish patients who underwent primary vascular surgical reconstruction, and for each ACEI user up to five nonusers were identified using propensity score matching followed by Cox regression. Cumulative risk of new vascular surgery in this cohort was $24.0 \%$ for ACEI users and $23.1 \%$ for nonusers (HR 1.21; 95\% CI 1.13-1.30). They found no differences regarding stroke and major amputation, but concluded that $\mathrm{ACE}$ use was associated with an increased long-term risk of recurrent vascular reconstruction. ${ }^{9}$

Shahin et al reviewed the evidence regarding the effects of ACEI in patients with symptomatic PAD of the lower limbs in terms of the effect on maximum and pain-free walking distances and ankle brachial pressure index. They reported that ACEI did not improve treadmill walking distance in patients with symptomatic lower limb arterial disease. ${ }^{10}$ Identifying patients undergoing intervention for varied symptomatology is important because the clinical course of PAD is dependent upon presenting symptoms. Historically, only $\sim 25 \%$ of patients with claudication will deteriorate to further limb ischemia, and very few, only $1 \%-3 \%$, need amputation within the first 5 years after initial diagnosis of claudication. ${ }^{11}$ This is, however, in contrast to the clinical course of patients presenting with CLI, which encompasses both rest pain and tissue loss/gangrene. Those who fall into this category generally have a poor prognosis for both life and limb. CLI patients at 1 year from diagnosis are known to have $-30 \%$ risk of amputation as well as a $25 \%$ risk of mortality. ${ }^{11}$ The very high risk of mortality at 1 year indicates severe systemic disease from comorbidities including hypertension, diabetes and its complications, as well as coronary disease.

\section{Limitations}

There are several limitations in our study. Medicare data are administrative and, as such, are limited by the coding systems. ICD-9-CM and CPT code data may not contain all the anatomic details and are limited by each coder and may vary between institutions. Also, all ACEIs are assumed to be equal in this study due to database constrictions. We also did not include any angiotensin-receptor blockers (ARBs), which are also now making headway in the market for patients unable to tolerate ACEI. However, other articles have suggested that due to the difference in receptor blockade by ARBs, wound healing may not be an issue. ${ }^{12}$ As an observational study, ACEI therapy was not randomly assigned, which could introduce selection bias. However, we were able to control patient demographics and comorbidities.

\section{Conclusion}

Overall, crude amputation rates were lower for patients who did not take an ACEI compared to those who took an ACEI before LER at 90 days and 1 year after procedure. Multivariable analysis after being adjusted for age, gender, race, disease severity, type of surgical procedure, and comorbidities did not show any association between ACEI use and overall poorer limb salvage rates. However, when the cohort was stratified by indication for the procedure, patients utilizing ACEI undergoing LER for CLI with a diagnosis of rest pain had amputation rates which were significantly higher compared to non-users which remained significant after adjustment at 1 year. More research are needed to define the pharmacologic interactions in these complex pathways and further evaluation is needed regarding optimal medical management of patients with CLI who use ACEI.

\section{Acknowledgment}

This abstract was presented at the 44th Annual Symposium of the Society for Clinical Vascular Surgery, March 12-16, 2016, Las Vegas, NV, USA. The abstract from the meeting is available via the following link: http://symposium.scvs.org/ abstracts/2016/MP61.cgi.

\section{Disclosure}

The authors report no conflicts of interest in this work.

\section{References}

1. Criqui MH, Denenberg JO, Langer RD, Fronek A. The epidemiology of peripheral arterial disease: importance of identifying the population at risk. Vasc Med. 1997;2(3):221-226. 
2. Roger VL, Go AS, Lloyd-Jones DM, et al. Heart disease and stroke statistics - 2011 update: a report from the American Heart Association. Circulation. 2011;123(4):e18-e209.

3. Beckwith C, Munger MA. Effect of angiotensin-converting enzyme inhibitors on ventricular remodeling and survival following myocardial infarction. Ann Pharmacother. 1993;27(6):755-766.

4. Yusuf S, Sleight P, Pogue J, Bosch J, Davies R, Dagenais G; Heart Outcomes Prevention Evaluation Study Investigators. Effects of an angiotensin-converting-enzyme inhibitor, ramipril, on cardiovascular events in high-risk patients. $N$ Engl J Med. 2000;342(3):145-153.

5. Ahimastos AA, Walker PJ, Askew C, et al. Effect of ramipril on walking times and quality of life among patients with peripheral artery disease and intermittent claudication: a randomized controlled trial. JAMA. 2013;309(5):453-460.

6. Ahimastos AA, Dart AM, Lawler A, Blombery PA, Kingwell BA. Reduced arterial stiffness may contribute to angiotensin-converting enzyme inhibitor induced improvements in walking time in peripheral arterial disease patients. J Hyperten. 2008;26(5):1037-1042.

7. Ahimastos AA, Latouche C, Natoli AK, Reddy-luthmoodoo M, Golledge J, Kingwell BA. Potential vascular mechanisms of ramipril induced increases in walking ability in patients with intermittent claudication. Cir Res. 2014;114(7):1144-1155.
8. Fujiyama S, Matsubara H, Nozawa Y, et al. Angiotensin AT(1) and AT(2) receptors differentially regulate angiopoietin-2 and vascular endothelial growth factor expression and angiogenesis by modulating heparin binding-epidermal growth factor (EGF)-mediated EGF receptor transactivation. Circ Res. 2001;88(1):22-29.

9. Hogh A, Lindholt JS, Nielsen H, Jensen LP, Johnsen SP. Use of angiotensin-converting enzyme inhibitors and cardiovascular outcomes following primary vascular surgery: a nationwide propensity score matched follow-up study. Vasc Endovascular Surg. 2012;46(7): 515-523.

10. Shahin Y, Mazari F, Chetter I. Do angiotensin converting enzyme inhibitors improve walking distance in patients with symptomatic lower limb arterial disease? A systematic review and meta-analysis of randomised controlled trials. Int J Surg. 2011;9(3):209-213.

11. Norgren L, Hiatt WR, Dormandy JA, Nehler MR, Harris KA, Fowkes FGR; TASC II Working Group. Inter-society consensus for the management of peripheral arterial disease (TASC II). Eur J Vasc Endovasc Surg. 2007;(33 Suppl 1):S1-S75.

12. Margolis DJ, Hoffstad O, Thom S, et al. The differential effect of angiotensin-converting enzyme inhibitors and angiotensin receptor blockers with respect to foot ulcer and limb amputation in those with diabetes. Wound Repair Regen. 2010;18(5):445-551.
Vascular Health and Risk Management

\section{Publish your work in this journal}

Vascular Health and Risk Management is an international, peerreviewed journal of therapeutics and risk management, focusing on concise rapid reporting of clinical studies on the processes involved in the maintenance of vascular health; the monitoring, prevention and treatment of vascular disease and its sequelae; and the involvement of

\section{Dovepress}

metabolic disorders, particularly diabetes. This journal is indexed on PubMed Central and MedLine. The manuscript management system is completely online and includes a very quick and fair peer-review system, which is all easy to use. Visit http://www.dovepress.com/ testimonials.php to read real quotes from published authors. 